\title{
FINITE LIFETIME EIGENFUNCTIONS OF COUPLED SYSTEMS OF HARMONIC OSCILLATORS
}

\author{
L. BOULTON ${ }^{1}$, S.A.M. MARCANTOGNINI ${ }^{2}$ AND M.D. MORÁN ${ }^{3}$
}

\begin{abstract}
We consider a Hermite-type basis for which the eigenvalue problem associated to the operator $H_{A, B}:=B\left(-\partial_{x}^{2}\right)+A x^{2}$ acting on $L^{2}\left(\mathbb{R} ; \mathbb{C}^{2}\right)$ becomes a three-terms recurrence. Here $A$ and $B$ are $2 \times 2$ constant positive definite matrices. Our main result provides an explicit characterization of the eigenvectors of $H_{A, B}$ that lie in the span of the first four elements of this basis when $A B \neq B A$.
\end{abstract}

\section{INTRODUCTION}

It is well known that the spectrum of the harmonic oscillator Hamiltonian

$$
H_{\alpha}:=-\partial_{x}^{2}+\alpha^{2} x^{2}, \quad \alpha>0
$$

acting on $L^{2}(\mathbb{R})$ consists of the non-degenerate eigenvalues $\{\alpha(2 n+1)\}_{n=0}^{\infty}$ with corresponding normalized eigenfunctions

$$
\phi_{n}^{\alpha}(x)=\frac{\alpha^{1 / 4} h_{n}\left(\alpha^{1 / 2} x\right) e^{-\alpha x^{2} / 2}}{\sqrt{2^{n} n ! \sqrt{\pi}}},
$$

where $h_{n}(x):=(-1)^{n} e^{x^{2}} \partial_{x}^{n}\left[e^{-x^{2}}\right]$ is the $n$-th Hermite polynomial. The present paper is devoted to studying the spectrum of a matrix version of $H_{\alpha}$, the operator

$$
H_{A, B}:=B\left(-\partial_{x}^{2}\right)+A x^{2},
$$

acting on $L^{2}\left(\mathbb{R} ; \mathbb{C}^{2}\right)$, where $A$ and $B$ are two $2 \times 2$ constant positive definite matrices.

In contrast to the scalar situation, the spectral analysis of $H_{A, B}$ is far more involved due to the non-commutativity of the coefficients. If $A B=B A$, it is not difficult to find the eigenvalues and eigenfunctions of $H_{A, B}$ from those of $H_{\alpha}$. On the other hand, when $A B \neq B A$, the

Date: March 2004.

2000 Mathematics Subject Classification. Primary: 34L40 ; Secondary: 34L10, 81Q10.

Key words and phrases. Non-commutative harmonic oscillators, higher dimensional Hermite basis, eigenfunction expansion. 
eigenvalues and eigenfunctions of $H_{A, B}$ are connected to those of $H_{\alpha}$ in a highly non-trivial manner (see Theorem 3 below).

Our recent interest in describing spectral properties of operators such as $H_{A, B}$ arises from two sources. In a series of recent works, Parmeggiani and Wakayama, cf. [4], [5] and [6], characterize the spectrum of the operator $K_{\tilde{A}, \tilde{B}}:=\tilde{A}\left(-\partial_{x}^{2}+x^{2}\right)+\tilde{B}\left(2 x \partial_{x}+x^{2}\right)$ acting on $L^{2}\left(\mathbb{R} ; \mathbb{C}^{2}\right)$, assuming that $\tilde{A}$ is definite positive and $\tilde{B}=-\tilde{B}^{t}$. Although the two operators are related, it does not seem possible to obtain the eigenvalues of $H_{A, B}$ from those of $K_{\tilde{A}, \tilde{B}}$. In [5] and [6] the eigenfunctions of $K_{\tilde{A}, \tilde{B}}$ are found in terms of a twisted Hermite-type basis of $L^{2}\left(\mathbb{R} ; \mathbb{C}^{2}\right)$. In this basis the eigenvalue problem associated to $K_{\tilde{A}, \tilde{B}}$ becomes a three-term recurrence. The strategy presented below for analyzing the spectrum of $H_{A, B}$ will be similar.

Our second motivation is heuristic. It is known that the scalar harmonic oscillator, $H_{\alpha}$, achieves the optimal value for the constant in the Lieb-Thirring inequalities with power $\sigma \geq 3 / 2$, cf. [2]. It would be of great interest finding Hamiltonians with similar properties for Lieb-Thirring-type inequalities for magnetic Schrödinger and Pauli operators, cf. [1], 2] and [3]. Due to their close connection with the harmonic oscillator, both $K_{\tilde{A}, \tilde{B}}$ and the presently discussed $H_{A, B}$ are strong candidates for further investigations in this direction.

The plan of the paper is as follows. Section 2 is devoted to describing elementary facts about $H_{A, B}$. In section 3 we consider a basis for which the eigenvalue problem associated to $H_{A, B}$ is expressed as a three-term recurrence. The main results are to be found in section 4 where we establish necessary and sufficient conditions, given explicitly in terms of the entries of $A$ and $B$, for an eigenfunction of $H_{A, B}$ to be the linear combination of the first four elements of this basis.

\section{Elementary PROperties of $H_{A, B}$}

We define $H_{A, B}$ rigorously as the self-adjoint operator whose domain, denoted below by $\mathcal{D}$, is the set of all

$$
\left(\begin{array}{c}
\phi \\
\psi
\end{array}\right) \in L^{2}\left(\mathbb{R} ; \mathbb{C}^{2}\right)
$$

such that

$$
\phi, \psi \in H^{2}(\mathbb{R}) \cap\left\{f \in L^{2}(\mathbb{R}): \int\left|x^{2} f(x)\right|^{2}<\infty\right\}=H^{2}(\mathbb{R}) \cap \widehat{H^{2}}(\mathbb{R}),
$$


where $H^{2}(\mathbb{R})$ denotes the Sobolev space of index $(2,2)$ and ". " denotes Fourier transform. Since

$$
\left\langle H_{A, B}\left(\begin{array}{c}
\phi \\
\psi
\end{array}\right),\left(\begin{array}{l}
\phi \\
\psi
\end{array}\right)\right\rangle=\int\left[B\left(\begin{array}{c}
\phi^{\prime} \\
\psi^{\prime}
\end{array}\right) \cdot\left(\begin{array}{c}
\phi^{\prime} \\
\psi^{\prime}
\end{array}\right)+A\left(\begin{array}{c}
x \phi \\
x \psi
\end{array}\right) \cdot\left(\begin{array}{c}
x \phi \\
x \psi
\end{array}\right)\right] \mathrm{d} x \geq 0,
$$

$H_{A, B}$ is a symmetric operator. It is well known (cf. 7]) that if the domain of $H_{\alpha}$ is chosen to be $H^{2}(\mathbb{R}) \cap \widehat{H^{2}}(\mathbb{R})$, then $H_{\alpha}$ is self-adjoint, non-negative and $\mathcal{S}(\mathbb{R})$, the Schwartz space, is a core for $H_{\alpha}$. Thus $H_{A, B}$ with domain $\mathcal{D}$ is a self-adjoint non-negative operator with core $\mathcal{S}\left(\mathbb{R} ; \mathbb{C}^{2}\right)$. Indeed, these properties are obvious when $A$ is a diagonal non-negative matrix and $B=\mathrm{Id}$, the identity matrix. The general case follows by considering the factorization

$$
H_{A, B}=B^{1 / 2} E^{*} H_{C, \text { Id }} E B^{1 / 2},
$$

where $B^{-1 / 2} A B^{-1 / 2}=E^{*} C E$ is the Jordan diagonalization of the former matrix, and by using the fact that $\mathcal{D}$ is invariant under the action of constant matrices.

Lemma 1. The spectrum of $H_{A, B}$ consists exclusively of isolated eigenvalues of finite multiplicity whose only accumulation point is $+\infty$. Moreover, if $\lambda_{n}$ denotes the $n$-th eigenvalue of this operator counting multiplicity, then

$$
a_{1}^{1 / 2} b_{1}^{1 / 2}(2 n+1) \leq \lambda_{2 n+1} \leq \lambda_{2 n+2} \leq a_{2}^{1 / 2} b_{2}^{1 / 2}(2 n+1),
$$

where $0<a_{1} \leq a_{2}$ and $0<b_{1} \leq b_{2}$, are the eigenvalues of $A$ and $B$, respectively.

Proof. It reduces to showing that

$$
\lambda_{n}\left(H_{A_{1}, B_{1}}\right) \leq \lambda_{n}\left(H_{A, B}\right) \leq \lambda_{n}\left(H_{A_{2}, B_{2}}\right),
$$

where $A_{j}=a_{j}(\mathrm{Id})$ and $B_{j}=b_{j}(\mathrm{Id})$. This follows directly from the min-max principle (cf. [8]), the estimates

$0<\left(\begin{array}{cc}a_{1} & 0 \\ 0 & a_{1}\end{array}\right) \leq A \leq\left(\begin{array}{cc}a_{2} & 0 \\ 0 & a_{2}\end{array}\right)$ and $0<\left(\begin{array}{cc}b_{1} & 0 \\ 0 & b_{1}\end{array}\right) \leq B \leq\left(\begin{array}{cc}b_{2} & 0 \\ 0 & b_{2}\end{array}\right)$,

and (2).

The above universal bound is not sharp in general and for most pairs $(A, B), \lambda_{2 n+1} \neq \lambda_{2 n+2}$.

As we mentioned earlier, it is not difficult to compute the eigenvalues and eigenfunctions of $H_{A, B}$ when $A$ and $B$ commute. Indeed $A B=B A$ if, and only if, $A$ and $B$ have one (and hence both) eigenvectors in common. Let $w_{j} \neq 0$ be such that $A w_{j}=a_{j} w_{j}$ and $B w_{j}=b_{j} w_{j}$, 
for $j=1,2$. Let $\phi_{n}^{\alpha}(x)$ be, as in (11), the eigenfunctions of $H_{\alpha}$. Let $\beta_{j}=\sqrt{a_{j} / b_{j}}>0$. Then

$$
\begin{aligned}
H_{A, B} w_{j} \phi_{n}^{\beta_{j}}(x) & =\left(-b_{j} \partial_{x}^{2}+a_{j} x^{2}\right) w_{j} \phi_{n}^{\beta_{j}}(x) \\
& =b_{j}\left(-\partial_{x}^{2}+\left(a_{j} / b_{j}\right) x^{2}\right) w_{j} \phi_{n}^{\beta_{j}}(x) \\
& =b_{j}^{1 / 2} a_{j}^{1 / 2}(2 n+1) w_{j} \phi_{n}^{\beta_{j}}(x) .
\end{aligned}
$$

By choosing $\left\|w_{j}\right\|=1$, the family $\left\{w_{j} \phi_{n}^{\beta_{j}}(x): j=1,2 ; n=0,1, \ldots\right\}$ is an orthonormal basis of $L^{2}\left(\mathbb{R} ; \mathbb{C}^{2}\right)$, hence

$$
\text { Spec } H_{A, B}=\left\{b_{j}^{1 / 2} a_{j}^{1 / 2}(2 n+1): j=1,2 ; n=0,1, \ldots\right\} .
$$

The analysis below will show that finding the eigenvalues and eigenfunctions of $H_{A, B}$ whenever $A B \neq B A$ is by no means of the trivial nature as the above case.

\section{Hermite expansion of the Eigenfunctions in the NON-COMMUTATIVE CASE}

Without further mention, we will often suppress the sub-indices in operator expressions. The structure of $H_{A, B} \equiv H$ allows us to decompose $L^{2}\left(\mathbb{R} ; \mathbb{C}^{2}\right)$ into two invariant subspaces where the eigenvalue problem can be studied independently. We perform this decomposition as follows. Given $\alpha>0$, let

$$
\begin{gathered}
\mathcal{H}_{+}^{\alpha}:=\operatorname{Span}\left\{v x^{j} e^{-\alpha x^{2} / 2}: v \in \mathbb{C}^{2}, j=2 k, k=0,1, \cdots\right\}, \\
\mathcal{H}_{-}^{\alpha}:=\operatorname{Span}\left\{v x^{j} e^{-\alpha x^{2} / 2}: v \in \mathbb{C}^{2}, j=2 k+1, k=0,1, \cdots\right\},
\end{gathered}
$$

and denote by $H^{ \pm}=H \mid\left(\mathcal{D} \cap \mathcal{H}_{ \pm}^{\alpha}\right)$. Since $\mathcal{H}_{ \pm}^{\alpha}$ are invariant under $\partial_{x}^{2}$, multiplication by $x^{2}$ and action of constant matrices, these spaces are also invariant under $H$. Hence $H^{ \pm}: \mathcal{D} \cap \mathcal{H}_{ \pm}^{\alpha} \longrightarrow \mathcal{H}_{ \pm}^{\alpha}$ are self-adjoint operators and

$$
\text { Spec } H=\operatorname{Spec} H^{+} \cup \operatorname{Spec} H^{-} \text {. }
$$

Let

$$
L=2^{-1 / 2}\left(x+\partial_{x}\right) \quad \text { and } \quad L^{*}=2^{-1 / 2}\left(x-\partial_{x}\right)
$$

be the annihilation and creation operators for the scalar harmonic oscillator. Then

$$
L \phi_{0}^{1}=0, \quad L \phi_{n}^{1}=n^{1 / 2} \phi_{n-1}^{1} \quad \text { and } \quad L^{*} \phi_{n}^{1}=(n+1)^{1 / 2} \phi_{n+1}^{1} .
$$

From these relations one can easily deduce the recurrent identities

$$
\begin{aligned}
& 2 \alpha x^{2} \phi_{n}^{\alpha}=(n+2)^{1 / 2}(n+1)^{1 / 2} \phi_{n+2}^{\alpha}+(2 n+1) \phi_{n}^{\alpha}+n^{1 / 2}(n-1)^{1 / 2} \phi_{n-2}^{\alpha}, \\
& 2 \alpha^{-1} \partial_{x}^{2} \phi_{n}^{\alpha}=(n+2)^{1 / 2}(n+1)^{1 / 2} \phi_{n+2}^{\alpha}-(2 n+1) \phi_{n}^{\alpha}+n^{1 / 2}(n-1)^{1 / 2} \phi_{n-2}^{\alpha},
\end{aligned}
$$


where, here and elsewhere, any quantity with negative sub-index is zero. Since $\left\{\phi_{n}^{\alpha}\right\}_{n=0}^{\infty}$ is an orthonormal basis for $L^{2}(\mathbb{R})$, we can expand any vector of $L^{2}\left(\mathbb{R} ; \mathbb{C}^{2}\right)$ via

$$
\left(\begin{array}{l}
\phi \\
\psi
\end{array}\right)=\sum_{n=0}^{\infty} v_{n} \phi_{n}^{\alpha}
$$

for a suitable unique sequence $\left(v_{n}\right) \in l^{2}\left(\mathbb{N} ; \mathbb{C}^{2}\right)$. Moreover, denoting by $N_{\alpha}:=\alpha^{-1} A-\alpha B$ and $M_{\alpha}:=\alpha^{-1} A+\alpha B$,

$$
\begin{aligned}
H\left(\begin{array}{l}
\phi \\
\psi
\end{array}\right)= & {\left[\left(-\partial_{x}^{2}\right) B+x^{2} A\right] \sum_{n=0}^{\infty} v_{n} \phi_{n}^{\alpha} } \\
= & \sum_{n=0} B v_{n}\left(-\partial_{x}^{2}\right) \phi_{n}^{\alpha}+A v_{n} x^{2} \phi_{n}^{\alpha} \\
= & \frac{1}{2} \sum_{n=0}(n+2)^{1 / 2}(n+1)^{1 / 2} N_{\alpha} v_{n} \phi_{n+2}^{\alpha}+(2 n+1) M_{\alpha} v_{n} \phi_{n}^{\alpha}+ \\
& +n^{1 / 2}(n-1)^{1 / 2} N_{\alpha} v_{n} \phi_{n-2}^{\alpha} \\
= & \frac{1}{2} \sum_{k=0}\left[k^{1 / 2}(k-1)^{1 / 2} N_{\alpha} v_{k-2}+(2 k+1) M_{\alpha} v_{k}+\right. \\
& \left.+(k+2)^{1 / 2}(k+1)^{1 / 2} N_{\alpha} v_{k+2}\right] \phi_{k}^{\alpha} .
\end{aligned}
$$

Thus $2 H^{ \pm}$are, respectively, similar to the block tri-diagonal matrices

$$
\left(\begin{array}{cccc}
S_{0}^{ \pm} & T_{1}^{ \pm} & & \\
T_{1}^{ \pm} & S_{1}^{ \pm} & T_{2}^{ \pm} & \\
& T_{2}^{ \pm} & S_{2}^{ \pm} & T_{3}^{ \pm} \\
& & T_{3}^{ \pm} & \ddots
\end{array}\right)
$$

acting on $l^{2}\left(\mathbb{N} ; \mathbb{C}^{2}\right)$, where

$$
\begin{gathered}
S_{k}^{+}=(4 k+1) M_{\alpha}, \quad T_{k}^{+}=(2 k)^{1 / 2}(2 k-1)^{1 / 2} N_{\alpha}, \\
S_{k}^{-}=(4 k+3) M_{\alpha} \quad \text { and } \quad T_{k}^{-}=(2 k)^{1 / 2}(2 k+1)^{1 / 2} N_{\alpha} .
\end{gathered}
$$

In order to reduce the amount of notation in our subsequent discussion, we consider $H_{A, B}$ in canonical form as follows. If $0 \leq b_{1} \leq b_{2}$ are the eigenvalues of $B$, let $U^{*}\left(\operatorname{diag}\left[b_{1}, b_{2}\right]\right) U$ be the diagonalization of $B$, and set $\tilde{A}:=b_{1}^{-1} U A U^{*}$ and $\tilde{B}:=\operatorname{diag}\left[1, b_{2} / b_{1}\right]$. Then

$$
H_{A, B}=b_{1} U^{*} H_{\tilde{A}, \tilde{B}} U
$$

where

$$
\tilde{A}=\left(\begin{array}{ll}
a & \xi \\
\bar{\xi} & c
\end{array}\right) \quad \text { and } \quad \tilde{B}=\left(\begin{array}{ll}
1 & 0 \\
0 & b
\end{array}\right)
$$


Here the positivity of $A$ and $B$ is equivalent to the conditions

$$
b \geq 1, \quad a, c>0 \quad \text { and } \quad 0 \leq|\xi|^{2}<a c .
$$

Furthermore notice that $A B=B A$ if, and only if, either $b=1$ or $\xi=0$. Hence, unless otherwise specified, we will consider without loss of generality that the pair $(A, B)$ is always the pair $(\tilde{A}, \tilde{B})$ in (15).

By virtue of the tri-diagonal representation (41), it seems natural to expect that the Hermite series (3) may be a good candidate for expanding the eigenfunctions of $H$. Not to mention that it is the obvious extension of the scalar and commutative cases. In this respect, we may consider "finite lifetime" series expansions of eigenfunctions $\Phi$ of $H$,

$$
\Phi=\sum_{n=0}^{k} v_{n} \phi_{n}^{\alpha(k)}
$$

for suitable finite $k \in \mathbb{N} \cup\{0\}, \alpha(k)>0$ and $v_{n} \in \mathbb{C}^{2}$. The results we present below show that, contrary to the above presumption, (3) is not such a good candidate for expanding $\Phi$ for small values of $k$. To be more precise, we show that for $k=0,1,2,3$, an expansion of type (6) is allowed only for a small sub-manifold of the region

$$
R:=\left\{(b, a, c,|\xi|) \in \mathbb{R}^{4}: a, c>0, b \geq 1,0 \leq|\xi|^{2}<a c\right\}
$$

corresponding to all positive definite pairs $(A, B)$.

We first discuss the cases $k=0,1$ and leave $k=2,3$ for the forthcoming section. The following result includes a family of test bases larger than the one considered in (6).

Lemma 2. Let $\phi_{n}^{\alpha}(x)$ be the eigenfunctions of the scalar harmonic oscillator $H_{\alpha}$. Then $H_{A, B}$ has an eigenfunction of the type $\Phi(x)=$ $\left(\tilde{a} \phi_{n}^{\alpha}(x), \tilde{b} \phi_{m}^{\beta}(x)\right)^{\mathrm{t}}$ where $\tilde{a}, \tilde{b} \in \mathbb{C}, \alpha, \beta>0$ and $m, n \in \mathbb{N} \cup\{0\}$, if and only if $A B=B A$.

Proof. If $H \Phi=\lambda \Phi$, then

$$
\begin{aligned}
& -\tilde{a}\left(\phi_{n}^{\alpha}\right)^{\prime \prime}+a x^{2} \tilde{a} \phi_{n}^{\alpha}+\xi x^{2} \tilde{b} \phi_{m}^{\beta}-\lambda \tilde{a} \phi_{n}^{\alpha}=0, \\
& -b \tilde{b}\left(\phi_{m}^{\beta}\right)^{\prime \prime}+c x^{2} \tilde{b} \phi_{m}^{\beta}+\bar{\xi} x^{2} \tilde{a} \phi_{n}^{\alpha}-\lambda \tilde{b} \phi_{m}^{\beta}=0 .
\end{aligned}
$$

If $\tilde{a}=0$ or $\tilde{b}=0$ in the above identities, necessarily $\xi=0$ so $A B=B A$. Hence without loss of generality we can assume that $\tilde{a} \tilde{b} \neq 0$.

If $\alpha \neq \beta$, once again $\xi=0$. Then we may suppose that $\alpha=\beta$. Since both left hand sides of the above identities are equal to $p(x) e^{-\alpha x^{2} / 2}$, where in both cases $p(x)$ is a polynomial of degree $2+\max (m, n)$, 
necessarily either $\xi=0$ or $m=n$. In the latter case, the above system is rewritten as

$$
\begin{aligned}
& -\tilde{a}\left(\phi_{n}^{\alpha}\right)^{\prime \prime}+(a \tilde{a}+\xi \tilde{b}) x^{2} \phi_{n}^{\alpha}-\lambda \tilde{a} \phi_{n}^{\alpha}=0, \\
& -b \tilde{b}\left(\phi_{n}^{\alpha}\right)^{\prime \prime}+(c \tilde{b}+\bar{\xi} \tilde{a}) x^{2} \phi_{n}^{\alpha}-\lambda \tilde{b} \phi_{n}^{\alpha}=0 .
\end{aligned}
$$

Since $\phi_{n}^{\alpha}$ is an eigenfunction of $H_{\alpha}$ where $\alpha>0$, necessarily $\xi \in \mathbb{R}$. Furthermore,

$$
\begin{gathered}
a+\xi \tilde{b} / \tilde{a}=\alpha^{2}=c / b+\xi \tilde{a} /(b \tilde{b}) \quad \text { and } \\
a+\xi \tilde{b} / \tilde{a}=\lambda /(2 n+1)=b c+\xi \tilde{a} b / \tilde{b} .
\end{gathered}
$$

Hence necessarily $b=1$.

Since $\mathcal{H}^{ \pm}$are invariant under the action of $H$, and the even (resp. odd) terms in the series (6) belong to $\mathcal{H}^{+}$(resp. $\mathcal{H}^{-}$), the above lemma ensures that $\Phi=v_{0} \phi_{0}^{\alpha}+v_{1} \phi_{1}^{\alpha}$ is an eigenfunction of $H$ if, and only if, $A$ and $B$ commute.

\section{Four-TERm Expansion of EIGENFunctions of $H_{A, B}$}

In this section we study necessary and sufficient conditions in order to guarantee that $\Phi \in L^{2}\left(\mathbb{R} ; \mathbb{C}^{2}\right)$, with finite lifetime expansion of the type (6) for $k=2$ and 3, is an eigenfunction of $H$ for suitable $\alpha(k)>0$ when $A B \neq B A$. In other words, assuming that $\Phi$ satisfies the constraint

$$
\Phi=v_{0} \phi_{0}^{\alpha}+v_{1} \phi_{1}^{\alpha}+v_{2} \phi_{2}^{\alpha}+v_{3} \phi_{3}^{\alpha},
$$

we aim to investigate conditions ensuring $H \Phi=\lambda \Phi$.

Since

$$
v \phi_{2 n}^{\alpha} \in \mathcal{H}_{+}^{\alpha} \quad \text { and } \quad v \phi_{2 n+1}^{\alpha} \in \mathcal{H}_{-}^{\alpha}, \quad v \in \mathbb{C}^{2}
$$

for all $n \in \mathbb{N} \cup\{0\}$, and the subspaces $\mathcal{H}_{ \pm}^{\alpha}$ are invariant under $H$, we may consider the even and odd cases separately. To this end, let

$$
\Phi^{+}=v_{0} \phi_{0}^{\alpha}+v_{2} \phi_{2}^{\alpha} \quad \text { and } \quad \Phi^{-}=v_{1} \phi_{1}^{\alpha}+v_{3} \phi_{3}^{\alpha},
$$

$\Phi^{ \pm} \in H^{ \pm}$respectively. Then our goal is to find necessary and sufficient conditions, given in terms of $(b, a, c,|\xi|) \in R \backslash \partial R$, ensuring that $\Phi^{ \pm}$is an eigenfunction of $H^{ \pm}$. The following is our main result.

Theorem 3. Let

$$
B=\left(\begin{array}{ll}
1 & 0 \\
0 & b
\end{array}\right) \quad \text { and } \quad A=\left(\begin{array}{ll}
a & \xi \\
\bar{\xi} & c
\end{array}\right)
$$

where $b>1, a, c>0$ and $0<|\xi|^{2}<a c$. Let $\beta>0$ be such that $\beta^{2}$ is an eigenvalue of $B^{-1 / 2} A B^{-1 / 2}>0$. Let

$$
\lambda_{\text {even }}:=\frac{5 \beta\left(a b+c-2 \beta^{2} b\right)}{a+c-(b+1) \beta^{2}} \quad \text { and } \quad \lambda_{\text {odd }}:=\frac{7 \beta\left(a b+c-2 \beta^{2} b\right)}{a+c-(b+1) \beta^{2}} .
$$


Then

i) $H^{+}$has an eigenfunction $\Phi^{+}(x)$ of type (8) if and only if

$$
2 \lambda_{\text {even }}\left[a+c-(b+1) \beta^{2}\right]=5 \beta\left(\beta-\lambda_{\text {even }}\right)\left(\lambda_{\text {even }}-\beta b\right) .
$$

In this case $\alpha=\beta$ and $H^{+} \Phi^{+}=\lambda_{\text {even }} \Phi^{+}$. Furthermore, $\lambda_{\text {even }}$ is an eigenvalue of $M_{\beta}$.

ii) $H^{-}$has an eigenfunction $\Phi^{-}(x)$ of type (8) if and only if

$$
6 \lambda_{\text {odd }}\left[a+c-(b+1) \beta^{2}\right]=7 \beta\left(3 \beta-\lambda_{\text {odd }}\right)\left(\lambda_{\text {odd }}-3 \beta b\right) .
$$

In this case $\alpha=\beta$ and $H^{-} \Phi^{-}=\lambda_{\text {odd }} \Phi^{-}$. Furthermore $\lambda_{\text {odd }}$ is an eigenvalue of $3 M_{\beta}$.

Notice that the conditions on $a, b, c$ and $\xi$ ensure that $A B \neq B A$.

Proof. Put

$$
\Phi^{+}(x)=\left(u_{0}+u_{2} x^{2}\right) e^{-\alpha x^{2} / 2},
$$

for $u_{0}, u_{2} \neq 0$. Since $H^{+}$is similar to the tri-diagonal matrix (4), then $H^{+} \Phi^{+}=\lambda \Phi^{+}$, if and only if

$$
\begin{aligned}
& S_{0}^{+} u_{0}+T_{1}^{+} u_{2}=\lambda u_{0} \\
& T_{1}^{+} u_{0}+S_{1}^{+} u_{2}=\lambda u_{2} \\
& T_{2}^{+} u_{2}=0 .
\end{aligned}
$$

The latter equation implies that $u_{2} \in \operatorname{ker} N_{\alpha}$ and thus the first one implies that $\lambda$ is an eigenvalue of $S_{0}^{+}=M_{\alpha}$ with associated eigenfunction $u_{0}$. A straightforward computation shows that the above system is equivalent to

$$
\begin{aligned}
& \left(A-\alpha^{2} B\right) u_{2}=0 \\
& \left(A-\alpha^{2} B\right) u_{0}+(5 \alpha B-\lambda) u_{2}=0 \\
& (\alpha B-\lambda) u_{0}-2 B u_{2}=0 .
\end{aligned}
$$

The first equation holds if and only if $\alpha=\beta$. Here

$$
\beta=+\sqrt{\frac{a b+c \pm \sqrt{(c-a b)^{2}+4|\xi|^{2} b}}{2 b}}
$$

and $u_{2}=\left(\begin{array}{c}c-\beta^{2} b \\ -\bar{\xi}\end{array}\right)$. Notice that in this case

$$
0=\operatorname{det}\left(A-\beta^{2} B\right)=\left(a-\beta^{2}\right)\left(c-\beta^{2} b\right)-|\xi|^{2} .
$$

Let $\tilde{u}_{2}=\left(\begin{array}{c}a-\beta^{2} \\ \xi\end{array}\right)$. Then $\tilde{u}_{2} \perp u_{2}$ and

$$
\left(A-\beta^{2} B\right) \tilde{u}_{2}=\left(a+c-(1+b) \beta^{2}\right) \tilde{u}_{2} .
$$




\section{Decompose}

$$
u_{0}=\gamma u_{2}+\tilde{\gamma} \tilde{u}_{2}
$$

for suitable $\gamma, \tilde{\gamma} \in \mathbb{C}$. Then the second identity of (11) holds if and only if, $\lambda=\lambda_{\text {even }}$ and

$$
\tilde{\gamma}\left(a+c-(1+b) \beta^{2}\right)^{2}\left(a-\beta^{2}\right)=5 \beta(1-b)|\xi|^{2} .
$$

The third identity of (11) can be rewritten as the system

$$
\begin{aligned}
& \gamma(\beta-\lambda)\left(c-\alpha^{2} b\right)+\tilde{\gamma}(\beta-\lambda)\left(a-\beta^{2}\right)=2\left(c-\beta^{2} b\right) \\
& \gamma(\beta b-\lambda)(-\bar{\xi})+\tilde{\gamma}(\beta b-\lambda)(\xi)=-2 b \bar{\xi}
\end{aligned}
$$

in $\gamma$ and $\tilde{\gamma}$. By finding $\tilde{\gamma}$ from this system (for instance by Newton's method) and by equating to (13), a straightforward computation yields (9).

The proof of ii) is similar.

Notice that there is a duality of signs in the definition of $\beta$ (cf. (12) ), so conditions (9) and (10) comprise two possibilities each. Let

$$
\Omega_{\text {even }}^{ \pm}:=\{(b, a, c,|\xi|) \in R: \text { (9) holds }\}
$$

and

$$
\Omega_{\text {odd }}^{ \pm}:=\{(b, a, c,|\xi|) \in R: \text { (10) holds }\},
$$

where the sign for the super-index is chosen in concordance to the sign in expression (12). By computing the partial derivatives of both sides of identities (9) and (10), a straightforward but rather long computation which we omit in the present discussion, shows that these four regions are smooth 3-manifolds embedded in $R \subset \mathbb{R}^{4}$, see (17).

The fact that $\Omega_{\text {even }}^{ \pm}$are non empty is consequence of the following observation. By fixing $\tilde{b}>1$ and $|\tilde{\xi}|>0$, and by putting $c=a \tilde{b}$, condition (9) can be rewritten as

$$
\begin{aligned}
0 & =\tilde{b}^{-1}(\tilde{b}+1)^{2}\left[2\left(a \tilde{b}+c-2 \beta^{2} \tilde{b}\right)-(\beta-\lambda)(\lambda-\tilde{b} \beta)\right] \\
& = \pm \frac{4|\tilde{\xi}|}{\sqrt{\tilde{b}}}(\tilde{b}+1)^{2}-\beta^{2}(1-9 \tilde{b})(9-\tilde{b}),
\end{aligned}
$$

where $\beta^{2}=a \pm|\tilde{\xi}| \tilde{b}^{-1 / 2}$. Then $(\tilde{b}, \tilde{a}, \tilde{a} \tilde{b},|\tilde{\xi}|) \in \Omega_{\text {even }}^{+}$whenever

$$
\tilde{a}=-\frac{|\tilde{\xi}|\left(5 \tilde{b}^{2}-90 \tilde{b}+5\right)}{\tilde{b}^{1 / 2}\left(9 \tilde{b}^{2}-82 \tilde{b}+9\right)}
$$

for $9<\tilde{b}<9+4 \sqrt{5}$, and $(\tilde{b}, \tilde{a}, \tilde{a} \tilde{b},|\tilde{\xi}|) \in \Omega_{\text {even }}^{-}$whenever

$$
\tilde{a}=\frac{|\tilde{\xi}|\left(5 \tilde{b}^{2}-90 \tilde{b}+5\right)}{\tilde{b}^{1 / 2}\left(9 \tilde{b}^{2}-82 \tilde{b}+9\right)}
$$



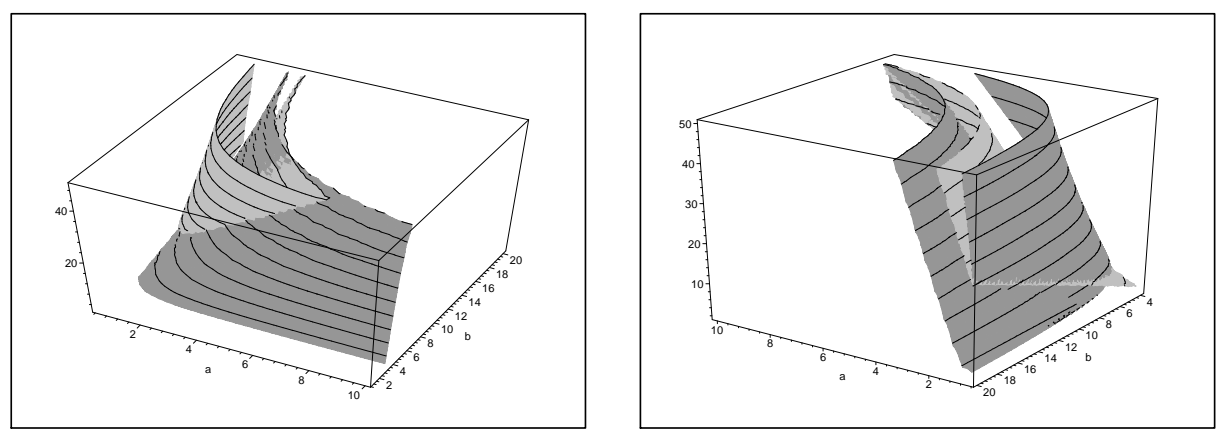

FiguRE 1. Left: projection of $\Omega_{\text {even }}^{+}$and $\Omega_{\text {even }}^{-}$onto the hyperplane $|\xi|=1$, the darker color corresponds to $\Omega_{\text {even }}^{+}$. Right: projection of $\Omega_{\text {odd }}^{+}$and $\Omega_{\text {odd }}^{-}$onto the hyperplane $|\xi|=1$. The lighter color corresponds to $\Omega_{\text {odd }}^{+}$.

for $1<\tilde{b}<9$ or $\tilde{b}>9+4 \sqrt{5}$. Furthermore, notice that if $(\tilde{b}, \tilde{a}, \tilde{a} \tilde{b},|\tilde{\xi}|) \in$ $\Omega_{\text {even }}^{ \pm}$, then

$$
(\tilde{b}, r \tilde{a}, r \tilde{c}, r|\tilde{\xi}|) \in \Omega_{\text {even }}^{ \pm}, \quad \text { for all } \quad r>0 .
$$

This shows that $\Omega_{\text {even }}^{ \pm}$are non-empty, unbounded and the semi-axis $\{(b, 0,0,0): b>1\}$ intersects $\partial \Omega_{\text {even }}^{ \pm}$. All these properties also hold for $\Omega_{\text {odd }}^{ \pm}$.

Furthermore,

$$
\Omega_{\text {even }}^{+} \cap \Omega_{\text {odd }}^{+}=\varnothing \quad \text { and } \quad \Omega_{\text {even }}^{-} \cap \Omega_{\text {odd }}^{-}=\varnothing .
$$

Indeed, if (9) and (10) hold at the same time for the same tetrad $(b, a, c,|\xi|)$, then, according to Theorem 3, $\lambda$ should be at the same time eigenvalue of $M_{\beta}$ and $3 M_{\beta}$ ( $\beta$ defined with the same sign). Obviously the latter is a contradiction. In figure 1 we reproduce the projections of these four regions, onto the hyper-plane $|\xi|=1$. These picture suggest that $\Omega^{+} \cap \Omega^{-} \neq \varnothing$.

Finally, we may comment on the issue of considering a more general basis for expanding the eigenfunctions of $H$. One might think that a natural candidate for generalizing (6) is the finite expansion

$$
\Phi=\sum_{j=0}^{m}\left(\begin{array}{c}
a_{j} \phi_{j}^{\alpha} \\
b_{j} \phi_{j}^{\beta}
\end{array}\right),
$$

where $a_{j}, b_{j}$ are complex number and $\alpha, \beta>0$. We studied a particular case of this in Lemma 2. It turns out that if $\Phi$ is an eigenfunction of the above form, then either $\xi=0$ or $\beta=\alpha$, so it should be as in (6). This can be easily proven by writing down the system for the eigenvalue 
equation and considering the asymptotic behaviour of the identities as $x \rightarrow \infty$.

\section{REFERENCES}

[1] L. Erdös, J.P. SoloveJ "Magnetic Lieb-Thirring inequalities with optimal dependence on the field strength". Preprint 2003, arXiv:math-ph/0306066.

[2] A. Laptev, T. Weide, "Recent results on Lieb-Thirring inequality", Université de Nantes. Exp. XX (2000) 1-14.

[3] A. Laptev, T. WeidL, "Sharp Lieb-Thirring inequalities in high dimensions", Acta Math. 184 (2000) 87-111.

[4] A. Parmeggiani, M. Wakayama, "Oscillator representations and systems of ordinary differential equations", Proc. Natl. Acad. Sci. USA 98 (2001) 26-30.

[5] A. Parmeggiani, M. Wakayama, "Non-commutative harmonic oscillators I", Forum Math. 14 (2002) 538-604.

[6] A. Parmeggiani, M. Wakayama, "Non-commutative harmonic oscillators II", Forum Math. 14 (2002) 669-690.

[7] M. Reed, B. Simon, Methods of modern mathematical physics, volume 2: self-adjointness, Academic press, New York, 1975.

[8] M. Reed, B. Simon, Methods of modern mathematical physics, volume 4: analysis of operators, Academic press, New York, 1978.

${ }^{1}$ LyOnell Boulton

Department of Mathematics

and Statistics,

University of Calgary,

2500 University Drive,

Calgary, AB, Canada T2N 1N4

email: Iboulton@math.ucalgary.ca

${ }^{3}$ María Dolores Morán

Escuela de Matemáticas,

Facultad de Ciencias,

Universidad Central de Venezuela,

Apartado 20513,

Caracas, 1020A, Venezuela.

email: mmoran@euler.ciens.ucv.ve
${ }^{2}$ Stefania Marcantognini

Departamento de Matemáticas, Instituto Venezolano de Investigaciones Científicas, Apartado 21827, Caracas, 1020A, Venezuela. email: smarcant@ivic.ve 